\title{
Design Thinking: creatividad y pensamiento crítico en la universidad
}

\section{Design Thinking: Creativity and Critical Thinking in College}

Cecilia Latorre-Cosculluela (*) https://orcid.org/0000-0002-6083-8759

Sandra Vázquez-Toledo (*) https://orcid.org/0000-0003-2206-2299

Ana Rodríguez-Martínez (*) https://orcid.org/0000-0002-9140-2974

Marta Liesa-Orús (*) https://orcid.org/0000-0002-9685-8399

(*) Universidad de Zaragoza

(Recibido: 15 de marzo de 2019; Aceptado para su publicación: 2 de mayo de 2019)

Cómo citar: Latorre-Cosculluela, C., Vázquez-Toledo, S., Rodríguez-Martínez, A. y Liesa-Orús, M. (2020). Design Thinking: creatividad y pensamiento crítico en la universidad. Revista Electrónica de Investigación Educativa, 22, e28, 1-13. https://doi.org/10.24320/redie.2020.22.e28.2917

\section{Resumen}

El modelo educativo propuesto desde el Espacio Europeo de Educación Superior requiere una transformación orientada hacia un aprendizaje socio-constructivista desde el que se prepare al alumnado para la globalización, el avance tecnológico y la capacidad de innovación constante. A este respecto, la utilización del enfoque metodológico Design Thinking está cobrando cada vez mayor relevancia en el proceso de formación universitaria de los futuros docentes. Desde esta perspectiva, se describe una experiencia de Design Thinking cuya finalidad es el diseño de un proyecto de innovación educativa y se analizan, mediante un cuestionario, las percepciones de 107 estudiantes universitarios sobre dicho proceso. Los resultados indican que los equipos de trabajo lograron diseñar planteamientos innovadores a problemáticas reales de su entorno comprometiéndose activamente en el proceso compartido de búsqueda de soluciones. Esta metodología activa supone un impulso a la confianza de los estudiantes en sus capacidades creativas y desarrollo de las habilidades empáticas.

Palabras clave: Pensamiento crítico, innovación, creatividad, Design Thinking.

\section{Abstract}

The educational model proposed by the European Higher Education Area calls for a transformation geared toward socio-constructivist learning that prepares students for globalization, technological advancement, and a capacity for constant innovation. In this regard, the design thinking methodological approach is becoming increasingly relevant in future teachers' college education. It is in this context that this study describes a design thinking experience aimed at designing an educational innovation project and uses a questionnaire to analyze 107 college students' perceptions of the process. The results show that the work teams were able to design innovative approaches to real problems they faced, becoming actively engaged in a shared search for solutions. This active methodology boosts students' confidence in their creative capacities and the development of empathic skills. 


\section{Introducción}

Durante los últimos años la investigación empírica ha evidenciado la existencia de diferentes prácticas docentes que tienen el enorme potencial de estimular una participación más activa y comprometida del alumnado. La consecuencia de algunos de estos procesos es que se logra potenciar el pensamiento creativo, crítico e innovador, la autonomía y el aprendizaje de distintas competencias transversales, profesionales y actitudinales por parte de los estudiantes (Almerich et al., 2018; González-González, 2014). Cada vez más profesionales de la educación a lo largo de las distintas etapas educativas no dudan en afirmar la importancia de ayudar a los estudiantes a desarrollar un modo de pensar más creativo y crítico fundamentado en la comprensión e ideación de representaciones innovadoras susceptibles de hacerse realidad (Bezanilla et al., 2018; Brown y Kuratko, 2015; Steinbeck, 2011). Esta capacidad de innovar se posiciona, incluso, como una de las habilidades cruciales del siglo XXI para una sociedad que se encuentra en progresiva evolución y cambio (Scheer et al., 2012; Wrigley y Straker, 2017; Wright y Wrigley, 2017).

Desafortunadamente, autores como Plucker et al. (2004) y Scheer et al. (2012) consideran que -entre los responsables de implementar estas prácticas en las aulas de nuestros sistemas educativos- se aprecian niveles preocupantes de desmotivación para la adopción de prácticas de enseñanza fundamentadas en el socio-constructivismo y la realización de procesos holísticos. Scheer et al. (2012) reflejan muy bien esta idea apuntando que:

Hay un eslabón perdido en la transferencia de los hallazgos teóricos de la ciencia de la pedagogía a la implementación práctica, lo que lleva a los profesores a focalizarse sobre prácticas que nada tienen que ver con el aprendizaje constructivista. (p. 10)

El presente estudio se propone con la finalidad de aportar evidencia empírica que avale la eficacia de algunas de estas aproximaciones metodológicas socio-constructivistas en auge en el siglo XXI. En concreto, se describe y analiza una experiencia fundamentada en el Pensamiento de Diseño (Design Thinking). Desde un punto de vista teórico se revisa la literatura relacionada con los constructos e ideas fundamentales vinculadas al Design Thinking y sus aplicaciones en el ámbito educativo. Luego, son presentados los objetivos del estudio, el procedimiento seguido en la aplicación de la experiencia práctica y el tipo de metodología utilizada. El estudio finaliza con la exposición y análisis de los resultados obtenidos, y con la extracción de las correspondientes conclusiones y perspectivas de futuro, apostando, en definitiva, por una educación inclusiva en la que estén presentes tanto el cuerpo como la mente (Sánchez y López, 2019).

\subsection{Proceso de aplicación del Design Thinking}

Las competencias clave en la educación tratan, precisamente, de la capacitación en la resolución de conflictos y problemas de la vida real a través de procesos de análisis y evaluación de los mismos, y el posterior planteamiento de soluciones al respecto (Brown y Kuratko, 2015; Scheer et al., 2012). Es en esos ámbitos destinados a la resolución de problemas complejos y al aprendizaje experiencial en los que el Design Thinking tiene su mayor presencia (Beckman y Barry, 2007; Elsbach y Stigliani, 2018). Esta técnica se concibe, pues, como una modalidad de pensamiento que entremezcla el conocimiento y entendimiento de las circunstancias y entorno en el que surgen los retos, y la creatividad para proponer soluciones, con la capacidad racional de contrastar dichas respuestas con la realidad próxima (Mosely et al., 2018; Wrigley y Straker, 2017).

Si bien en sus inicios el Design Thinking se encontraba más vinculado a profesionales del ámbito del diseño, en la actualidad se identifica su relación con otras áreas, disciplinas y profesiones (Dijksterhuis y Silvius, 2017; Rauth et al., 2010; Wrigley et al., 2018). Este enfoque metodológico es considerado, por Mentzer et al. (2015) como un planteamiento de aprendizaje auténtico en tanto que, durante su desarrollo, se ponen en práctica predisposiciones básicas que se clasifican dentro de la perspectiva socio-constructivista del aprendizaje: la motivación para explorar nuevos territorios, la apertura a nuevas ideas y propuestas, el pensamiento creativo y, también, otro conjunto de competencias metacognitivas. Se da a los estudiantes 
la oportunidad de iniciar habilidades de autoexploración, compartir el conocimiento con los demás y mejorar competencias de trabajo en equipo, tales como la expresión asertiva de opiniones y la empatía.

Desde sus inicios, el Design Thinking se ha relacionado con los principios del aprendizaje experiencial, inicialmente popularizado por Kolb (1984). De acuerdo con este autor, el aprendizaje experiencial se entiende como un proceso de transformación de la experiencia que conduce a la creación de nuevos conocimientos, resultantes de la combinación de las acciones de percibir y modificar la experiencia. Por tanto, se aprende haciendo (Melles et al., 2015). En el Design Thinking la atención se concentra en su mayoría en el proceso de diseño de la solución, antes que en el producto final en sí mismo desde la formación de equipos multidisciplinares sólidos (Brown y Wyatt, 2010). En concreto, el Design Thinking:

(...) asume como necesidad unir el pensamiento racional y lógico con la intuición, vínculo que favorece un marco de trabajo que va más allá del pensamiento deductivo tradicional, enfocado, sobre todo, a proporcionar soluciones válidas, para abrazar un pensamiento abductivo orientado a soluciones que se han de explorar, no descubiertas o planteadas previamente. (Gasca, 2015, p. 23).

La puesta en práctica de esta aproximación metodológica se estructura en torno a un conjunto de fases dinámicas y definidas, pero no por ello del todo independientes (Cassim, 2013). Conforman, todas ellas, la naturaleza esencial del funcionamiento del Design Thinking. Brown (2008) denomina a estas fases secuenciales que combinan el descubrimiento con la ideación y prototipado, como "Inspiración, Ideación e Implementación". No obstante, otros autores han descrito estas fases con mayor detalle e incluso se han servido de diferentes términos. En cualquier caso, todas las versiones parten de una valoración previa del alcance del problema a resolver (con mayor o menor detalle), y conducen a la búsqueda de resultados en los que, necesariamente, deben tenerse en cuenta las opiniones de las personas implicadas en su resolución y otros posibles interesados. Por ello se considera condición indispensable comprender en profundidad la realidad y contexto de los sujetos para los que se van a diseñar y proponer las soluciones, y empatizar con ellos (Gasca, 2015).

\subsection{El Design Thinking para trabajar la innovación}

Dada la dispersión de ideas y la considerable cantidad de hipótesis con las que se trabaja en el ámbito de la innovación, la metodología Design Thinking se convierte en la estrategia ideal para la exploración de nuevas decisiones, el contraste de diferentes ideas y, en consecuencia, la toma de mejores decisiones (Castillo et al., 2014; González-González, 2014). En este proceso se trata de transformar por completo la perspectiva del pensamiento, dejando atrás el "diseño para personas" para comenzar a "diseñar con las personas" (Gasca, 2015). Puesto que se parte de una previa exposición de ideas, se rompen las posibles barreras comunicativas y mejoran exponencialmente las interacciones verbales y no verbales entre los participantes, es así como se logra potenciar el pensamiento innovador y desarrollar la creatividad (Wrigley y Straker, 2017).

La agencia de diseño IDEO y la Universidad de Standford fueron las instituciones pioneras en el estudio y aplicación del enfoque de Design Thinking, primero al ámbito de la innovación en el diseño, y después a la educación (Plattner et al., 2012). Desde la citada universidad se desarrolló el método como un enfoque independiente y transferible a partir de la consideración de los principios del diseño centrado en la persona Human-Centred Design (HCD) (Gasca, 2015; Melles et al., 2015). En tanto que el Design Thinking conduce a despertar la creatividad en las personas que trabajan bajo su enfoque supone un canal directo hacia los procesos de innovación y la proactividad. El participante se integra dentro de un proceso en el que es partícipe activo de la actividad innovadora (González-González, 2014).

\section{3 ¿Cómo se aplica el Design Thinking en la formación universitaria?}

Las posibilidades de desarrollar un pensamiento de diseño en estudiantes de diversos campos de conocimiento son ampliamente reconocidas por diferentes autores (Fischer, 2015; Renard, 2014; Wrigley et al., 2018). En la formación universitaria, en las disciplinas relacionadas con la ingeniería Dym et al. (2005) 
ofrecen evidencia de las mejoras que el Design Thinking produce sobre la capacidad de retención de conocimientos de los estudiantes, la satisfacción con sus propios aprendizajes y la diversidad de competencias y aprendizajes adquiridos. Por su parte, Benson y Dresdow (2014) proponen el uso de prácticas con Design Thinking para estructurar y definir el proceso de evaluación en el área de administración y educación empresarial. Los diseñadores de este proceso son los propios profesores, quienes participan en conversaciones acerca del aprendizaje de sus estudiantes realizando conexiones entre diferentes disciplinas.

A través de un estudio de caso, Lim et al. (2013) analizan las capacidades creativas de varios estudiantes universitarios de una asignatura de Diseño y Tecnología en la Universidad de Singapur. Utilizando una pedagogía facilitadora, por parte del docente, hacia el proceso de aprendizaje, cada estudiante logra proponer soluciones novedosas e innovadoras a los problemas de diseño que les son planteados. Por su parte, Cassim (2013) describe una experiencia con Design Thinking con estudiantes en formación para la creación de un proyecto en el área de Diseño. Los autores subrayan la relevancia de este enfoque metodológico en tanto que podría llegar a desempeñar un papel estratégico en la sociedad sudafricana reflejado en la mejora de sistemas de vivienda, transporte, reciclaje y salud, entre otras. Desde la aplicación de cursos en formato online, también se han implementado ciertas experiencias de desarrollo del pensamiento creativo en el alumnado. Sirve, a modo de ejemplo, el trabajo de Bower (2011), en el que el rediseño del entorno de una conferencia online conduce a una co-construcción del conocimiento entre el conjunto de estudiantes y el desarrollo de la capacidad de compartir su pensamiento de diseño.

La aplicación del Design Thinking sobre los campos de la pedagogía innovadora y otros espacios de aprendizaje ha recibido una limitada atención en las últimas décadas. De manera paulatina comienza en nuestros días a generar mayor entusiasmo, desde las primeras etapas educativas hasta las instituciones de Educación Superior (Retna, 2016). De hecho, y siguiendo lo expuesto por Steinbeck (2011), a la par que la metodología del Design Thinking cruza horizontes más amplios que aquel relacionado con sus aplicaciones en ingeniería o diseño, surge la necesidad de desarrollar estudios empíricos que evidencien los beneficios de su utilización en otras áreas y disciplinas.

Del conjunto de estudios mencionados que aluden al ámbito de la enseñanza universitaria, y tal como Renard (2014) sugiere, resulta destacable la diversidad de modalidades en las que el Design Thinking es interpretado e implementado. A este respecto, juegan en todos ellos un papel crucial la evaluación auténtica, la toma de decisiones basada en el pensamiento, la capacidad de retroalimentación entre los miembros de los grupos de trabajo y la autorreflexión sobre el proceso seguido y aprendizajes adquiridos.

Considerando las evidentes ventajas que, hasta el momento, la literatura ha demostrado con respecto a la incorporación de metodologías de aprendizaje activas en el aula universitaria, surge la necesidad de implementar y evaluar, desde las percepciones de los principales implicados, nuevas experiencias en las que se pongan en práctica enfoques de estas características. Por tanto, se propone este estudio con el objetivo de describir una experiencia de aprendizaje en el ámbito universitario consistente en diseñar y crear un proyecto de innovación educativa utilizando el enfoque de Design Thinking. Se establece como segundo objetivo la identificación de las percepciones de los estudiantes implicados en la experiencia, los resultados tras su aplicación y otras conclusiones asociadas a la resolución de la tarea. El estudio se enmarca en una de las asignaturas del primer cuatrimestre del primer curso del Grado de Magisterio en Educación Primaria de una universidad del sistema universitario español.

\section{Método}

El marco metodológico de esta investigación se sustenta en las características propias de los enfoques cuantitativo y cualitativo dentro de la metodología descriptiva. Se optó por ambas perspectivas porque responden al objetivo que se persigue. En palabras de Hernández et al. (2003), los estudios descriptivos permiten especificar las propiedades, características y perfiles importantes de personas, grupos o cualquier otro fenómeno que es sometido a un análisis. En este caso, va a describir el proceso de implementación de la metodología Design Thinking en el aula y su impacto en la formación del maestro. 
La muestra participante en el estudio estuvo conformada por 107 estudiantes universitarios del Grado de Magisterio en Educación Primaria del curso 2018-19. Del total de participantes, 56\% pertenece al género masculino y $44 \%$ al femenino, todos ellos se encontraban matriculados en el primer curso del mencionado grado universitario. La edad de los participantes se encuentra comprendida entre los 18 y los 25 años.

Para la recogida de datos se adaptó un cuestionario de 12 ítems con una escala Likert de 5 puntos (desde "totalmente en desacuerdo", a "totalmente de acuerdo"). Los ítems fueron extraídos del estudio de Reyes et al. (2018), en el que los autores analizan las percepciones de un conjunto de estudiantes universitarios acerca de una experiencia de aprendizaje utilizando la metodología de Flipped Classroom. Рara su construcción fueron consideradas variables tales como la metodología específica propuesta (Design Thinking) (ítems 3, 4, 6, 8, 9 y 10), los recursos y materiales educativos utilizados (ítems 2, 5, 7 y 11 ) y otros aspectos operativos (por ejemplo, las órdenes e instrucciones que recibieron los estudiantes, y el tiempo disponible para la realización de la experiencia) (ítems 1 y 12). En la sección final de la encuesta se añadieron dos preguntas abiertas en las que se preguntaba a los estudiantes por aquellos aspectos a destacar, y otros susceptibles de mejora en el desarrollo de la experiencia.

Previo a la presentación del proyecto de innovación educativa utilizando el Design Thinking, el profesorado de la asignatura seleccionó los materiales que iban a ser requeridos tanto para ofrecer a los estudiantes las explicaciones oportunas como para la implementación de la tarea durante la sesión presencial. Desde un primer momento quedó fijado el objetivo correspondiente a la tarea final a elaborar: "Diseñar y construir un proyecto creativo de innovación educativa que tenga la capacidad de responder a una problemática real detectada en un entorno concreto, aplicando metodologías activas que logren incluir a toda la comunidad educativa a la que va destinada dicho proyecto". Siguiendo la propuesta de Goldman et al. (2014), se dispuso una estructura de trabajo en equipos de aprendizaje cooperativo correspondiente al modo en que habitualmente desarrollan su trabajo en el aula.

La aplicación de la experiencia se realizó con base en las fases propuestas y definidas por Beckman y Barry (2007) y Steinbeck (2011): empatizar, definir, idear, construir prototipos y probar. Durante la fase empatizar se dedicó tiempo y energía al desarrollo de una lluvia de ideas sobre el problema con el propósito de que cada estudiante se sintiera partícipe del proceso. Este procedimiento contrasta, como apuntan Beckman y Barry (2007), con otros enfoques en los que se parte de un problema o proyecto bien definido desde un primer momento. Los equipos de estudiantes se sumergieron en un intenso proceso de desarrollo de empatía hacia diferentes problemáticas educativas de su entorno próximo. Con ello se buscaba una adquisición de conocimientos e información sobre los usuarios a los que se iba a destinar el proyecto de innovación educativa, así como empatizar con sus necesidades auténticas. Todo estuvo mediado por la explicación inicial del docente, quien acotó con exactitud que este análisis de necesidades de los entornos educativos debía estar fundamentado en una observación lo más objetiva posible teniendo en cuenta la realidad de los centros (motivación del alumnado, igualdad de género, utilización de metodologías activas, desarrollo de competencias emocionales).

Durante la fase definir,los estudiantes delimitaron con claridad la problemática específica a la que querían dar solución, considerando todas las necesidades detectadas en la primera etapa. El docente pautó los ritmos de trabajo y, previo al inicio de esta fase, explicó que la definición del problema debía precisarse en un solo enunciado, de la forma más breve posible. La fase idear se caracterizó por la generación y especificación de todas aquellas representaciones e ideas posibles acerca del reto educativo al que habían decidido hacer frente; de nuevo, se dieron las indicaciones pertinentes relacionadas con el tiempo del que disponían para delimitar estas ideas y acerca del modo en que debían ser especificadas en papel (concretas, claras y bien delimitadas). Posteriormente, los equipos de trabajo desarrollaron y construyeron un modeloprototipo del proyecto (figura 1) lo más próximo al definitivo, con las ideas más creativas y prometedoras surgidas durante la fase de ideación. 


\begin{tabular}{|c|c|c|c|c|}
\hline 1 & Retos a los que nos enfrentamos & Problemas & \multicolumn{2}{|c|}{ Público al que nos dirigimos } \\
\hline 2 & $\begin{array}{l}\text { Descripción de las competencias } \\
\text { que se abordan }\end{array}$ & $\begin{array}{l}\text { Principales } \\
\text { competencias }\end{array}$ & \multicolumn{2}{|c|}{ Otras competencias } \\
\hline 3 & Cómo se trabaja las competencias & Metodologías & $\begin{array}{l}\text { Indicadores de } \\
\text { desempeño }\end{array}$ & Postulados \\
\hline 4 & Productos obtenidos & Material realizado & \multicolumn{2}{|r|}{ Links } \\
\hline 5 & $\begin{array}{l}\text { Innovaciones pedagógicas } \\
\text { detectadas }\end{array}$ & & & \\
\hline
\end{tabular}

Figura 1. Elementos básicos que describen un proyecto innovador

Teniendo en cuenta la imposibilidad de llevar a la práctica los proyectos de innovación creados por estos estudiantes, la última fase (probar) fue sustituida por un proceso de evaluación del diseño que habían elaborado. Mediante la utilización de una rúbrica denominada "Diana de autoevaluación de un proyecto educativo innovador" (Observatorio Innovación para la Inclusión, 2014), pudieron identificar visualmente en qué medida cada uno de los planteamientos cumplía los mínimos de calidad en diferentes aspectos (utilización de metodologías activas, uso de la evaluación como proceso de aprendizaje, propuesta de experiencia de aprendizaje sostenible, vital y digital, aprendizaje más allá del aula).

Cada equipo de trabajo completó un ejemplar de las rúbricas de evaluación correspondiente al diseño de su proyecto de innovación educativa. A partir de esta rúbrica se derivaron los resultados que muestra la figura 2, expresados en términos de medias (teniendo en cuenta que puntuaban cada ámbito en una escala Likert de 1 a 4) para cada uno de los equipos. La mayor parte de los grupos de trabajo ha valorado el diseño de sus proyectos de innovación como experiencias que fomentan el aprendizaje colaborativo y un aprendizaje auténtico y vital. Por otro lado, se han detectado las carencias más notables en la inclusión de un aprendizaje digital, así como en el uso de la evaluación como herramienta de aprendizaje.

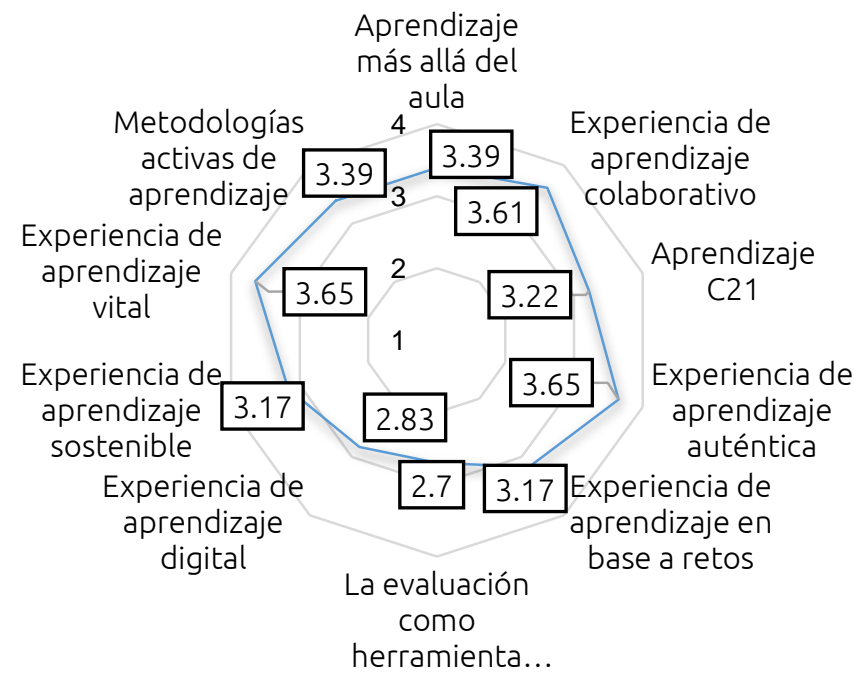

Figura 2. Resultados de las rúbricas de evaluación del proyecto de innovación educativa

El cuestionario fue enviado al alumnado a través de un enlace Web el mismo día en que los proyectos de innovación educativa fueron expuestos en el aula. Se les indicó, además de las instrucciones para su respuesta, el carácter voluntario de la cumplimentación del mismo. Una vez recogidos los datos se realizó el análisis estadístico de los resultados utilizando SPSS 22.0. En los resultados del estudio se presentan los 
valores promedio de las percepciones señaladas por los estudiantes universitarios en cada uno de los ítems valorados, así como los índices de desviación típica. En última instancia se sintetizó y analizó, por bloques de contenidos, la información cualitativa recabada de las preguntas abiertas que acompañaban al cuestionario.

\section{Resultados}

Las puntuaciones medias de las percepciones reportadas por los estudiantes de la muestra se presentan en la tabla I. En primer lugar, y respecto a la dimensión referida a la metodología utilizada (ver ítems 3, 4, $6,8,9$ y 10), la puntuación promedio obtenida $(M=4.19)$ indica que la experiencia ha resultado interesante a estos estudiantes. Destaca positivamente $(M=4.18)$ la utilidad del proyecto de innovación en su futura práctica profesional como docentes. Las percepciones de los estudiantes fueron también positivas en las variables relacionadas con un mejor entendimiento del concepto de innovación $(M=3.99)$, y en la importancia de las actividades previas preparatorias para el adecuado desarrollo de la actividad práctica (M $=4.03)$. Con la mayor media de puntuación obtenida $(M=4.45)$, destaca la buena consideración que ha tenido este grupo de estudiantes sobre la propuesta de crear su proyecto de innovación siguiendo los pasos de esta técnica del Design Thinking.

La segunda dimensión propuesta se encuentra vinculada a los materiales y recursos educativos empleados. Se aprecia, en primer lugar, un entendimiento general del concepto de "innovación educativa" gracias a los recursos utilizados en esta técnica de resolución de problemas a través de retos $(M=4.22)$. Resaltan las discrepancias entre las consideraciones de los estudiantes sobre la necesidad que han tenido de consultar recursos adicionales a los ofrecidos en el aula para construir la tarea final $(M=2.79)$, pues se ha obtenido una desviación típica de 1.18 puntos. Los recursos que les fueron presentados (mapa de empatía, diferentes materiales didácticos y visuales, construcción del póster final) también han resultado de gran interés y utilidad para los estudiantes $(M=4.31 ; M=4.19)$.

Los resultados obtenidos en la tercera dimensión analizada, correspondiente a aspectos operativos adicionales, sugieren la existencia de ligeros desacuerdos especialmente al respecto de la suficiencia del tiempo destinado al desarrollo de la actividad práctica en las sesiones presenciales $(M=3.23)$. Concretamente, se deriva una desviación estándar en este ítems de 1,15 puntos. Las instrucciones presentadas por los profesores han sido consideradas, por el contrario, suficientes y claras $(M=4.10)$.

Las puntuaciones medias otorgadas a los diferentes ítems del cuestionario son, en términos generales, considerablemente positivas. Cabe resaltar, por tanto, que esta aproximación metodológica a la resolución de retos de manera creativa, tiene una importante aceptación por parte de este conjunto de estudiantes universitarios con los que se ha realizado la experiencia. No obstante, resulta necesario considerar también las desviaciones estándar que, en cada uno de los ítems, se observan, pues es indicativo de la existencia de cierta variedad de opiniones entre la muestra de alumnos. 
Tabla I. Puntuaciones medias de las percepciones de los estudiantes

\begin{tabular}{|c|c|c|}
\hline & Media & $\begin{array}{l}\text { Desviación } \\
\text { estándar }\end{array}$ \\
\hline $\begin{array}{l}\text { P1_Las instrucciones dadas para el desarrollo del ejercicio práctico y las actividades } \\
\text { preparatorias han sido claras y suficientes. }\end{array}$ & 4.10 & .764 \\
\hline $\begin{array}{l}\text { P2_A través de los recursos propuestos pude entender el concepto de "Proyecto de } \\
\text { Innovación Educativa". }\end{array}$ & 4.22 & .731 \\
\hline $\begin{array}{l}\text { P3 Me ha resultado interesante la metodología propuesta (Design Thinking) para abordar } \\
\text { el Proyecto de Innovación. }\end{array}$ & 4.19 & .859 \\
\hline P4_El trabajo del Proyecto de Innovación será útil en mi práctica profesional. & 4.18 & .888 \\
\hline $\begin{array}{l}\text { P5_He necesitado consultar algunos otros recursos adicionales a los que la profe me } \\
\text { propuso, para entender el concepto de Innovación Educativa. }\end{array}$ & 2.79 & 1.180 \\
\hline $\begin{array}{l}\text { P6_Considero que las actividades de preparación han sido fundamentales para desarrollar } \\
\text { el ejercicio del Proyecto de Innovación. }\end{array}$ & 4.03 & .841 \\
\hline $\begin{array}{l}\text { P7_Me han resultado interesantes los recursos propuestos (mapa de empatía, materiales } \\
\text { utilizados, construcción de un póster para presentar el proyecto) para abordar la creación } \\
\text { del Proyecto de Innovación. }\end{array}$ & 4.31 & .706 \\
\hline $\begin{array}{l}\text { P8_Mi percepción acerca de la "Innovación Educativa" ha cambiado tras realizar la } \\
\text { actividad práctica propuesta. }\end{array}$ & 3.79 & 898 \\
\hline $\begin{array}{l}\text { P9_El diseño del Proyecto de Innovación me ha ayudado a entender mejor el concepto de } \\
\text { "Innovación Educativa". }\end{array}$ & 3.99 & .852 \\
\hline $\begin{array}{l}\text { P10_Me parece buena idea que hayamos pensado la temática sobre la que queríamos que } \\
\text { fuera nuestro Proyecto de Innovación Educativa, siguiendo los pasos de la metodología } \\
\text { de Design Thinking (empatizar, definir e idear). }\end{array}$ & 4.45 & .743 \\
\hline $\begin{array}{l}\text { P11_Los recursos propuestos (mapa de empatía, materiales utilizados, cuadro-estructura } \\
\text { de un proyecto de innovación de la Fundación Telefónica), son útiles para comprender el } \\
\text { concepto de Innovación Educativa. }\end{array}$ & 4.19 & .802 \\
\hline $\begin{array}{l}\text { P12_El tiempo durante las sesiones presenciales ha sido suficiente para el desarrollo de la } \\
\text { actividad práctica. }\end{array}$ & 3.23 & 1.154 \\
\hline
\end{tabular}

Del análisis de contenido de las respuestas ofrecidas a las cuestiones abiertas planteadas al final del cuestionario también se deriva información de interés para el estudio. A grandes rasgos, en la primera cuestión relacionada con las ventajas percibidas de la construcción del proyecto de innovación con Design Thinking, surgen seis grandes bloques de contenido que configuran las temáticas centrales alrededor de las que se sitúan las respuestas de los estudiantes: creatividad, implicación activa, cooperación, motivación, reflexión y aprendizaje duradero.

La ventaja más destacada que perciben los estudiantes del enfoque de Design Thinking es, con absoluta claridad, la posibilidad de desarrollo de sus habilidades creativas e imaginativas. Repara, la mayoría de ellos, en los beneficios que les proporciona no sólo al estimular su creatividad en la creación del proyecto de innovación, sino en su capacidad de expresarse y reflejar múltiples ideas y pensamientos. Ejemplo de ello se aprecia en el comentario una de las estudiantes:

Nos ha ayudado en la tarea de pensar nuevas ideas, fomentar nuestra creatividad en grupo y, también, a imaginar muchas más posibilidades en el momento de tener que confeccionar nuestro proyecto de innovación.

Otra de las cuestiones más destacadas de la experiencia está vinculada con el desarrollo de habilidades para cooperar con los miembros del equipo en aras de alcanzar un objetivo común. En una primera fase relacionada con la experiencia de "empatía", se comparten pensamientos, ideas, representaciones mentales en forma de imágenes y gráficos, y diferentes juicios y opiniones. Llevan a cabo un proceso de descubrimiento común en el que, siempre desde esta perspectiva cooperativa, ponen en marcha su capacidad investigadora. Debaten, discuten, van perfilando la idea sobre la que versará su proyecto de innovación y trabajan juntos considerando en todo momento las aportaciones de cada miembro del grupo, como señala uno de los estudiantes: 
Pienso que hemos conseguido realizar el proyecto de innovación educativa desde una identidad personal y particular como equipo de trabajo. Trabajamos juntos valorando las ideas de nuestros compañeros porque siempre valen. A partir de allí, intentamos llegar a una en común mucho más elaborada que si sólo pensamos en la nuestra en concreto.

El hecho de no atender a una clase magistral para recibir la explicación sobre el tipo de proyecto de innovación a construir fue también varias veces mencionado. Los estudiantes destacan el aspecto motivador del Design Thinking, su capacidad para despertar el interés por aprender y el atractivo y atención que demanda elaborar un proyecto desde una perspectiva que, para ellos, resulta totalmente novedosa. Es el reto inicial de búsqueda de una problemática particular y real de su entorno el que los sumerge en un auténtico proceso de aprendizaje. Captan el estímulo, lo toman como un aliciente y, a partir de ese instante son las interacciones entre el equipo las que continúan manteniendo la motivación ante la tarea:

El Design Thinking es un tipo de metodología muy diferente a la tradicional con la que se consigue motivar al estudiante, pues resulta atractiva y llama la atención. Dado que trabajamos de manera interactiva y tenemos un reto inicial que resolver, prestamos mayor atención e interés. Nos motiva mucho más que la clase magistral. Además, si vamos a trabajar en un proyecto de innovación, qué mejor que recibir la explicación con una metodología innovadora.

En último lugar, cabe mencionar los beneficios que los estudiantes indican respecto a la capacidad reflexiva y la vivencia de un aprendizaje más duradero tras participar en esta experiencia. De este modo, son ellos mismos los que piensan y construyen su idea innovadora para responder a una determinada problemática de la sociedad, lo que les conduce a elaborar un proyecto de innovación con capacidad para mejorar la educación destinada a los niños del sistema educativo. Véase, como reflejo de ello, el siguiente comentario de una estudiante:

Al disponer de total libertad para debatir y proponer el tema del proyecto de innovación, nos permite reflexionar e investigar con más profundidad. Al implicarnos mucho más que en una clase tradicional, terminamos aprendiendo cuestiones que duran más a largo plazo que lo que estudiamos para el examen.

Si bien el alumnado no ha destacado serias desventajas de la utilización de este enfoque, dos estudiantes han sugerido algunas recomendaciones que merecen ser señaladas. Por un lado, subrayan la necesidad de destinar mayor tiempo a la preparación y construcción del proyecto de innovación contando con la tutoría del profesorado dentro del aula. Y, por otro, mencionan la posibilidad de que surjan pequeños conflictos derivados de las diferencias en las opiniones personales en el momento de compartir ideas. No obstante, la misma estudiante finaliza su argumento apuntando que "también es cierto que la variedad es positiva".

\section{Conclusiones}

En la etapa universitaria en la que se enmarca este estudio, la aplicación de este enfoque metodológico ha contribuido a que los integrantes de los equipos perciban el alcance de aprendizajes auténticos y significativos. De manera progresiva se aproximan a la solución final en función de sus experiencias, del contraste de opiniones y de los acuerdos e ideas surgidos en grupo. Uno de los aspectos más interesantes de este método es que se focaliza en la acción, en el "hacer", en pasar de lo teórico a lo práctico, en demostrar. Los equipos de trabajo han cumplido el objetivo de diseñar soluciones innovadoras a problemas reales involucrándose activamente en un proceso compartido de búsqueda de ideas creativas.

Los resultados del presente estudio muestran coincidencia con las afirmaciones recogidas por Renard (2014), quien destaca el valor intrínseco que adquieren las formas de desarrollo de conocimiento de diseño sobre diversas capacidades de los estudiantes. Entre ellas, resalta las habilidades de resolución de problemas reales, la capacidad de utilizar distintos modelos cognitivos y, también, el desarrollo de un pensamiento y una comunicación de carácter no verbal. Steinbeck (2011) no duda al afirmar que enfoques como el aquí presentado persiguen con creces la creatividad y la innovación en el ámbito de la enseñanza 
y el aprendizaje.

En otras áreas de conocimiento en las que se han implementado procesos con Design Thinking para la búsqueda de soluciones a determinadas problemáticas también se han hallado resultados favorables. Véase, a modo de ejemplo, el estudio de Cahn et al. (2016), en el que un grupo de profesionales de la salud logra consensuar una serie de recomendaciones para fijar líneas futuras de investigación en la evaluación de la educación interprofesional del personal sanitario. También en el trabajo de Lloyd (2013), en el que se aplica una encuesta a estudiantes y profesores tras participar en una experiencia "a distancia" con Design Thinking, se alcanzan niveles de éxito percibido notables.

Practicar el pensamiento de diseño en la etapa de formación universitaria tiene el enorme potencial de desarrollar la capacidad para avanzar por sendas de conocimientos poco definidas y actuar en consecuencia y acorde a contextos reales para lograr las transformaciones deseadas. Estas habilidades de pensamiento de orden superior les da a los estudiantes la posibilidad, además, de innovar, analizar, crear, sintetizar y, con base en ello y tal como exponen Razzouk y Shute (2012), responder a problemas reales de nuestra sociedad. Se le añade, a todo ello, que la metodología Design Thinking implica un desarrollo positivo en el manejo y la expresión de los espacios y el tiempo (Ferrari, 2017). De este modo, los universitarios activan su atención y favorecen el proceso de aprendizaje (Conesa-Ros y Angosto, 2017).

Lo hallado en este estudio supone una contribución adicional al cuerpo emergente de literatura sobre las aplicaciones del Design Thinking en la formación universitaria y, en particular, sobre el proceso de adquisición de competencias transversales y específicas del Grado de Magisterio. Estos estudiantes han logrado focalizar sus esfuerzos en despertar la empatía hacia las personas a las que el proyecto de innovación educativa iba dirigido, siguiendo un proceso similar al de los alumnos del estudio de Lam y Suen (2015). Resulta lógico, por tanto, que la práctica de la inteligencia emocional constituya una parte inherente al desarrollo del rendimiento creativo, como del Moral et al. (2018) apuntan en las conclusiones de su estudio. Han generado ideas verdaderamente creativas reflejadas en la construcción de proyectos destinados a la estimulación de las inteligencias múltiples de Howard Gardner, al desarrollo de la personalidad y la individualidad de los niños desde las etapas iniciales de escolarización, o a la gestión de las emociones mediante un proyecto al que denominaron "emoción(arte)", entre otras ideas.

El Design Thinking tiene el extraordinario poder de dejar en las manos de los estudiantes universitarios la responsabilidad ante lo que desean aprender, sin tener respuestas únicas. Se instaura, de este modo, un modelo centrado en el aprendizaje del estudiante que estimula su autonomía y habilidades de autorregulación (Gargallo et al., 2017). Despertemos, por tanto, la confianza de los estudiantes sobre su capacidad creativa, para que logren convertirse en agentes activos de sus propios aprendizajes. Trabajemos con su curiosidad y pensamiento crítico, de manera que se les permita proponer soluciones innovadoras de mejora social y educativa, y participar en la compleja sociedad actual.

\section{Referencias}

Almerich, G., Díaz, I., Cebrián, S. y Suárez, J. (2018). Estructura dimensional de las competencias del siglo XXI en alumnado universitario de educación. Revista Electrónica de Investigación y Evaluación Educativa, 24(1), 1-21. https://doi.org/10.7203/relieve.24.1.12548

Beckman, S. L. y Barry, M. (2007). Innovation as a learning process. Embeding Design Thinking. California Management Review, 50(1), 25-57. https://doi.org/10.2307/41166415

Benson, J. y Dresdow, S. (2014). Design thinking: a fresh approach for transformative assessment practice. Journal of Management Education, 38(3), 436-461. https://doi.org/10.1177/1052562913507571

Bezanilla, M. J., Poblete, M., Fernández, D., Arranz, S. y Campo, L. (2018). El pensamiento crítico desde la perspectiva de los docentes universitarios. Estudios Pedagógicos, 44(1), 89-113.

https://doi.org/10.4067/S0718-07052018000100089 
Bower, M. (2011). Redesigning a web-conferencing environment to scaffold computing students' creative design processes. Educational Technology \& Society, 14(1), 27-42.

Brown, T. (2008). Definitions of Design Thinking. https://designthinking.ideo.com/?p=49

Brown, T. J. y Kuratko, D. F. (2015). The impact of design and innovation on the future of education. Psychology of Aesthetics, Creativity, and the Arts, 9(2), 147-151. https://doi.org/10.1037/aca0000010

Brown, Y. y Wyatt, J. (2010). Design Thinking for Social Innovation. Stanford Social Innovation Review, 8(1), 31-35. https://doi.org/10.1596/1020-797X 12129

Cahn, P. S., Bzowyckyj, A., Collins, L., Dow, A., Goodell, K., Johnson, A. F., Klocko, D., Knab, M., Parker, K., Reeves, S. y Zierler, B. K. (2016). A design thinking approach to evaluating interprofessional education. Journal of Interprofessional Care, 30(3), 378-380. https://doi.org/10.3109/13561820.2015.1122582

Cassim, F. (2013). Hands on, hearts on, minds on: Design Thinking within an education context. International Journal of Art \& Design Education, 32(2), 190-202. https://doi.org/10.1111/j.1476-8070.2013.01752.x

Castillo, M., Álvarez, A. y Cabana, R. (2014). Design Thinking: cómo guiar a estudiantes, emprendedores y empresarios en su aplicación. Ingeniería Industrial, 35(3), 301-311.

Conesa-Ros, E. y Angosto, S. (2017). Análisis del contenido de «expresión corporal» en los planes de estudio de grado en ciencias de la actividad física y el deporte en universidades españolas. Journal of Sport and Health Research, 9(2), 263-272.

del Moral, M. E., Villalustre, L. y Neira, M. R. (2018). Percepción docente del desarrollo emocional y creativo de los escolares derivado del diseño colaborativo de Digital Storytelling. Educación XX1, 21(1), 345-374. https://doi.org/10.5944/educxx1.20202

Dijksterhuis, E. y Silvius, G. (2017). The Design Fhinking approach to projects. The Journal of Modern Project Management, 4(3), 33-41.

Dym, C., Agogino, A., Eris, O., Frey, D. y Leifer, L. (2005). Engineering design thinking, teaching, and learning. Journal of Engineering Education, 94(1), 103-120. https://doi.org/10.1002/j.2168-9830.2005.tb00832.x

Elsbach, K. D. y Stigliani, I. (2018). Design Thinking and organizational culture: a review and framework for future research. Journal of Management, 44(6), 2274-2306. https://doi.org/10.1177/0149206317744252

Ferrari, H. (2017). Marta Schinca, precursora del teatro del movimiento. Fundamentos.

Fischer, M. (2015). Design it! Solving sustainability problems by applying design thinking. Gaia, 24(3), 174178. https://doi.org/10.14512/qaia.24.3.9

Gargallo, B., Pérez, C. Verde, I. y García, E. (2017). Estilos de aprendizaje en estudiantes universitarios y enseñanza centrada en el aprendizaje. Revista Electrónica de Investigación y Evaluación Educativa, 23(2), 124. https://doi.org/10.7203/relieve.23.2.9078

Gasca, J. (2015). Design Thinking. Afrontar los retos con la actitud de un diseñador. Leaners Magazine, 8, 2225. http://www.leanersmag.com/docs/publicaciones/08-design-acting/design-thinking.pdf 
Goldman, S., Kabayadondo, Z., Royalty, A., Carroll, M. P. y Roth, B. (2014). Student teams in search of Design Thinking, En L. Leifer, H. Plattner y C. Meinel (Eds.), Design Thinking research: building innovation eco-systems (pp. 11-34). Springer.

González-González, C. (2014). Estrategias para trabajar la creatividad en la educación superior: pensamiento de diseño, aprendizaje basado en juegos y en proyectos. Revista de Educación a Distancia, 40, 2-15.

Hernández, R., Fernández, C. y Baptista, P. (2003). Tipos de investigación. McGraw Hill.

Kolb, D. (1984). Experiential learning as the science of learning and development. Prentice Hall.

Lam, Y. Y. y Suen, B. Y. S. (2015). Experiencing empathy in design education through community engagement. International Journal of Continuing Education and Lifelong Learning, 7(2), 53-69.

Lim, S. S. H., Lim-Ratnam, C. y Atencio, M. (2013). Understanding the processes behind student designing: cases from Singapore. Design and Technology Education: An International Journal, 18(1), 20-29.

Lloyd, P. (2013). Embedded creativity: teaching Design Thinking via distance education. International Journal of Technology and Design Education, 23(3), 749-765. https://doi.org/10.1007/s10798-012-9214-8

Melles, G., Anderson, N., Barrett, T. y Thompson-Whiteside, S. (2015). Problem finding through Design Thinking in Education. En P. Blessinger y J. M. Carfora (Eds.), Inquiry-based learning for multidisciplinary programs: a conceptual and practical resource for educators (pp. 189-208). Emerald Group Publishing Limited.

Mentzer, N., Farrington, S. y Tennenhouse, J. (2015). Strategies for teaching brainstorming in Design educations. International Technology Education Association, 74(8), 8-13.

Mosely, G., Wright, N. y Wrigley, C. (2018). Facilitating Design Thinking: a comparison of design expertise. Thinking Skills and Creativity, 27, 177-189. https://doi.org/10.1016/i.tsc.2018.02.004

Observatorio Innovación para la Inclusión (2014). Decálogo de un proyecto innovador: guía práctica Fundación Telefónica. https://observatorio.profuturo.education/blog/2014/09/12/decalogo-de-un-proyecto-innova dor-guia-practica-fundacion-telefonica/

Plattner, H., Meinel, C. y Liefer, L. (2012). Design Thinking research: studying co-creation in practice. Springer.

Plucker, J., Beghetto, R. A. y Dow, G. (2004). Why isn't creativity more important to educational psychologists? Potential, pitfalls, and future directions in creativity research. Educational Psychologist, 39(2), 83-96. https://doi.org/10.1207/s15326985ep3902 1

Rauth, I., Köppen, E., Jobst, B. y Meinel, C. (2010). Design Thinking: an educational model towards creative confidence. Actas de la 1a. Conferencia Internacional de Diseño y Creatividad ICDC 2010. Kobe, Japón. https://www.designsociety.org/publication/30267/Design+Thinking\%3A+An+Educational+Model+toward s+Creative+Confidence

Razzouk, R. y Shute, V. (2012). What is Design Thinking and why is it important? Review of Educational Research, 82(3), 330-348. https://doi.org/10.3102/0034654312457429

Renard, H. (2014). Cultivating Design Thinking in students through material inquiry. International Journal of Teaching and Learning in Higher Education, 26(3), 414-424. 
Retna, K. S. (2016). Thinking about "Design Thinking": a study of teacher experiences. Asia Pacific Journal of Education, 36(1), 5-19. https://doi.org/10.1080/02188791.2015.1005049

Reyes, A. M., Cañón, M. J. y Olarte, F. A. (2018). Una propuesta de aula invertida en la asignatura de señales y sistemas de la Universidad Nacional de Colombia. Revista Educación en Ingeniería, 13(25), 82-87.

Sánchez, G. y López, M. (2019). Análisis de los contenidos de expresión corporal impartidos en la formación inicial de los docentes de primaria. Educación XX1, 22(1), 425-447.

https://doi.org/10.5944/educxx1.20058

Scheer, A., Noweski, C. y Meinel, C. (2012). Transforming constructivist learning into action: Design Thinking in education. Design and Technology Education: An International Journal, 17(3), 8-19.

Steinbeck, R. (2011). El Design Thinking como estrategia de creatividad en la distancia. Comunicar, 37(19), 27-35. https://doi.org/10.3916/C37-2011-02-02

Wright, N. y Wrigley, C. (2017). Broadening design-led education horizons: conceptual insights and future research directions. International Journal of Technology and Design Education, 29, 1-23. https://doi.org/10.1007/s10798-017-9429-9

Wrigley, C. y Straker, K. (2017). Design Thinking pedagogy: the educational design ladder. Innovations in Education and Teaching International, 54(4), 374-385. https://doi.org/10.1080/14703297.2015.1108214

Wrigley, C., Mosely, G. y Tomitsch, M. (2018). Design Thinking education: a comparison of massive open online courses. The Journal of Design, Economics, and Innovation, 4(3), 275-292.

https://doi.org/10.1016/i.sheji.2018.06.002 